\title{
Design Principles for Broad-Spectrum Protein-Crystal Nucleants with Nanoscale Pits
}

\author{
Jacobus A. van Meel, ${ }^{1}$ Richard P. Sear, ${ }^{2}$ and Daan Frenkel ${ }^{3}$ \\ ${ }^{1}$ FOM Institute for Atomic and Molecular Physics, Science Park 104, 1098 XG Amsterdam, The Netherlands \\ ${ }^{2}$ Department of Physics, University of Surrey, Surrey GU2 7XH, United Kingdom \\ ${ }^{3}$ Department of Chemistry, Cambridge University, Lensfield Road, Cambridge CB2 1EW, United Kingdom
}

(Received 7 August 2010; published 8 November 2010)

\begin{abstract}
Growing high-quality crystals is a bottleneck in the determination of protein structures by x-ray diffraction. Experiments find that materials with a disordered pitted surface seed the growth of protein crystals. Here we report computer simulations of rapid crystal nucleation in nanoscale pits. Nucleation is rapid, as the crystal forms in pits that have filled with liquid via capillary condensation. Surprisingly, we find that pits whose surfaces are rough are better than pits with crystalline surfaces; the roughness prevents the growing crystal from trying to conform to the pit surface and becoming strained.
\end{abstract}

PACS numbers: 81.10.Aj, 64.60.Q-, 64.70.dg, 87.15.nt

The faster crystals grow, the more defects they contain. Hence, high-quality protein crystals that can be used in $\mathrm{x}$ ray or neutron diffraction studies are ideally grown at low supersaturation where crystal growth is slow. Yet, to nucleate a protein crystal from solution, the supersaturation cannot be too small, because then crystals never form as nucleation is effectively suppressed. The challenge for protein crystallization is therefore to nucleate crystals at supersaturations that are so low that crystal growth is slow. One possible strategy to achieve this objective is to make use of heterogeneous nucleation: a suitable nucleation agent can induce crystallization under conditions where homogeneous (bulk) crystal nucleation is negligible and crystal growth is slow. The design of suitable crystalnucleating agents is therefore of great practical importance.

The rational design of such agents is hampered by a lack of understanding of the molecular mechanism of nucleation. One might think that the best strategy is to use structured templates that match the lattice spacing of the target crystal. Indeed, experiments on colloidal systems have shown that a template with a periodic surface pattern can strongly enhance crystal growth via epitaxy [1]. However, unless there is a precise match between the lattice spacing of the template and that of the epitaxially grown crystal, epitaxial crystal growth is not possible. Thus templates tend to work only for specific target crystals that are commensurate with the periodicity of the template. Such highly specific templates are of little use if we want to crystallize a wide variety of proteins with different and a priori unknown crystal lattices.

An alternative to templates was pioneered by Chayen and co-workers [2-4], who showed that materials with disordered nanoscale pitted surfaces can act as "universal" nucleation agents in the sense that they induce the nucleation of crystals of many different proteins. See Refs. [5,6] for reviews of protein crystallization.

In this Letter we present Monte Carlo simulations that investigate the mechanism of protein crystallization in nanoscale pits. Our aim is to study heterogeneous crystal nucleation in a simple model system with a phase diagram similar to that of many globular-protein solutions, such as lysozyme. Our computer simulations reveal that the optimal nucleation mechanism has two steps: capillary condensation of a protein "liquid" followed by rapid crystal nucleation. The simulations also show that the nucleation rate has a counterintuitive dependence on the roughness and crystallinity of the surface of the pit. Only when the surface is rough and amorphous (and not crystalline) do crystals grow readily without stress or defects.

In our model system, particles interact via a spherically symmetric potential, and the particles have no internal degrees of freedom. This is clearly a simplification; the interactions between proteins are clearly more complex. However, our mechanism relies only on capillary condensation, which is a thermodynamic phenomenon independent of the details of the interactions. Also, nucleants with pitted surfaces induce the crystallization of a range of proteins [3,5], suggesting that details of the interactions are not important in experiment.

Our model particles interact via the potential introduced by ten Wolde and Frenkel [7], with their range parameter $\alpha=50$. This short-ranged potential is known to have a bulk phase diagram that is qualitatively the same as that of a number of solutions of globular proteins [7]. A key characteristic of such phase diagrams is the presence of a metastable dilute-solution-to-concentrated-solution transition that lies within the coexistence region of the dilute-solution-to-crystal transition [8]. Homogeneous nucleation has been studied for this model [7,9]. In our simulations the protein molecules are attracted by, and nucleate on, substrates that are of three types: amorphous, smooth, and crystalline.

In what follows, we use reduced units. We define the reduced distance as $r^{*}=r / \sigma$, where $\sigma$ is the diameter of the particles. The reduced temperature $T^{*}=k_{B} T \epsilon^{-1}$, where $\epsilon$ is the well depth of the particle-particle attraction, 
and the reduced density $\rho^{*}=\rho \sigma^{3}$. All quantities reported in this work are expressed in these reduced units. Therefore, we omit the superscript asterisk (*) from here on.

Here we work exclusively at a temperature a little below the critical temperature, $T=0.89 T_{c}=0.374$, and at a chemical potential $0.04 k T$ below the metastable vaporliquid transition, i.e., just outside the vapor-liquid transition. This is at a (vapor) density $\rho=0.04$. There, the crystal phase is more stable than the vapor (dilute solution) phase, as we are within the vapor-crystal coexistence region. The vapor is the next most stable, but as we are near vapor-liquid coexistence, the liquid is nearly as stable as the vapor phase. In the vapor phase but near a vapor-liquid transition, pits with surfaces that attract the particles fill with liquid via capillary condensation [10]. This occurs at a distance from the vapor-liquid transition that scales inversely with the pit radius [10].

We performed Metropolis Monte Carlo simulations in the grand-canonical ensemble, where the temperature, volume, and chemical potential are kept constant, and the particle number is allowed to fluctuate [11]. This ensures a constant fluid pressure and minimizes finite size effects. For the interaction between a smooth surface and the particles, we use the same functional form as for the particle-particle interaction. Our crystalline and amorphous surfaces are made from fixed (i.e. immobile) particles which also interact with the protein particles via a potential of the same form and with the same diameter $\sigma$ but with well depth $\epsilon_{w}$. For the computation of free-energy barriers, we use umbrella sampling with a bias on the number of particles in the largest crystalline cluster, as described by ten Wolde et al. [12]. Crystalline particles are identified with bond-order parameters [13].

Figure 1 shows cross sections of simulation snapshots for nucleation in a cylindrical pit [Fig. 1(a)] and at a wall [Fig. 1(b)]; both have rough amorphous surfaces. The particles that form the pit or wall (shown in gray) are immobile yet otherwise identical to the "protein" particles. The concentrated disordered protein phase (blue particles) — which we call the protein "liquid"-fills the pit in Fig. 1(a) via capillary condensation. Then, in the liquidfilled pit, a crystal (yellow particles) nucleates, and eventually grows out of the pit. At a wall, a thin layer of protein liquid is adsorbed and a crystal can nucleate, too. Note, however, that the diameter of a critical crystal nucleus exceeds the width of the liquid layer.

Capillary condensation is where liquid fills a pit before it is stable in the bulk, i.e., when the vapor is the stable phase in the bulk [10]. The free energy of a small pit has a relatively large contribution from the surface term. This is proportional to $\gamma_{p v}$ for an empty pit and $\gamma_{p l}$ for a liquidfilled pit; $\gamma_{p v}$ and $\gamma_{p l}$ are the interfacial tensions for the interfaces between the pit surface and the vapor, and between the pit surface and the liquid, respectively. If $\gamma_{p l}<\gamma_{p v}$, small pits will fill with liquid via capillary condensation, as this reduces the surface term in the pit
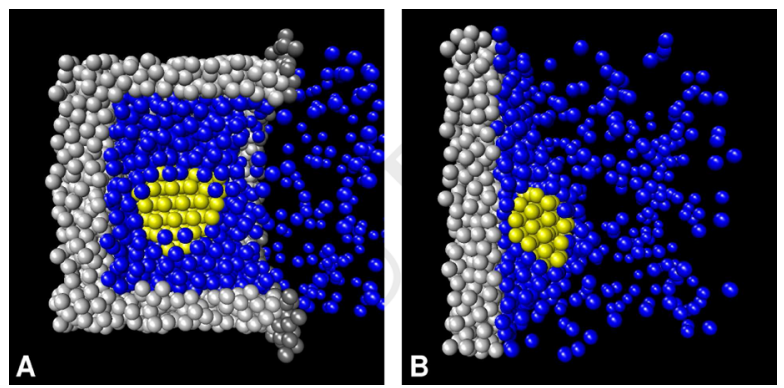

FIG. 1 (color online). Simulation snapshots of crystallization. The snapshots show cross sections through our simulation box. The surfaces are made of fixed particles that are equal in size to the model protein particles. Fixed particles that attract the model protein particles are light gray $\left(\epsilon_{w}=\epsilon\right)$; fixed particles that are repulsive are dark gray. The model protein particles themselves are shown in blue and yellow. Blue particles are in a locally liquidlike environment, while yellow particles are in a locally crystalline environment. Panel (A) is a snapshot of nucleation in a droplet of liquid in a pit with rough walls. Note the layer of liquid particles (blue) between the yellow growing crystal and the wall. Panel (B) is a snapshot of nucleation occurring at a flat rough surface with a thin adsorbed liquid layer.

free energy. In our simulations the pit fills spontaneously, without a nucleation barrier.

In Fig. 2(a) we have plotted the computed nucleation barrier height $\Delta G^{*}$ in pits with rough amorphous surfaces as a function of pit radius $R$. We find that there is a clear minimum in the nucleation barrier at pore radii that are small enough to allow capillary condensation yet large enough to allow the crystal nucleus to form in them. Note that this minimum has a different origin from the minimum in the nucleation barrier found by Page and Sear [14] for nucleation of a fluid from a pore. However, in both cases materials with a range of pit sizes, as found on disordered surfaces, are advantageous because on such surfaces there will always be some pits that are just the right size and shape.

It is perhaps counterintuitive that nucleation is favored by pits bounded by a rough inner surface. After all, a rough, disordered surface suppresses crystal growth in contact with the surface of the pit. However, the nucleating crystal then "floats" in the pit, separated from the walls by a thin layer of liquid. As the crystal nucleus is fully immersed in liquid, the nucleation barrier is much lower than in the bulk vapor, $25 k_{B} T$ not $80 k_{B} T$ as it is in the vapor. On a planar disordered surface the nucleus is only partially immersed [see Fig. 1(b)], and the barrier is intermediate between its value in the vapor and in the pit [see Fig. 2(b)].

As the nucleus is not in direct contact with the wall [see Fig. 1(a) and 1(b)], there is no requirement for the lattice of the nucleus to match the structure of the wall; the nucleation barrier is universally low-regardless of the lattice constant or symmetry of the crystal phase. We stress that although a low barrier is obtained for all crystal lattices, a nearby liquid-liquid transition is required. Such transitions 

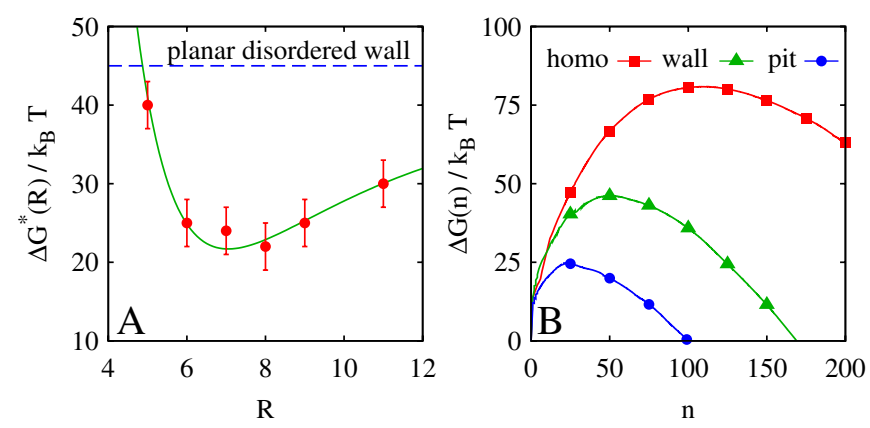

FIG. 2 (color online). (A) The free-energy barrier to nucleation, $\Delta G^{*}$, as a function of the radius of the pit, $R$, in units of the protein diameter $\sigma$. The pit sides are made of fixed particles with the same diameter as the model protein and so are rough on the length scale of the protein. (B) The nucleus free energy, $\Delta G$, as a function of the number of particles, $n$, in the nucleus. Shown are curves for homogeneous nucleation, heterogeneous nucleation on a wall with a rough surface, and heterogeneous nucleation in a pit with optimal size (radius $R=8$, depth $D=9$ ).

are common in protein solutions $[15,16]$, and crystallization often occurs near these transitions [17]. The present results provide a possible rationale for the common observation of protein crystallization near liquid-liquid phase separation [17].

So far we have discussed results for pits with surfaces with a roughness that is comparable to the size of the model protein molecules. In what follows, we examine the effect of surface roughness and consider crystalline surfaces, where epitaxy is possible.

First, we focus on the effect of varying the surface roughness of a wall or pit. As the surface is made smooth, the liquid orders at the surface. We studied cylindrical pits with flat bottoms [18]. We found that at the curved inner surface of the cylinder, the particles ordered but that this ordering is different from the ordering in a bulk crystal. This ordering along the inside of the cylindrical surface competes with the ordering at the flat bottom of the pore, and it is different from and not compatible with bulk crystalline ordering. Once this incompatible ordering forms, it inhibits crystallization. Thus we do not see rapid crystallization for these smooth-walled pits.

In contrast to smooth-walled pits, a completely flat, smooth wall does induce crystallization, and we find rapid nucleation. A flat (but not a curved) wall induces layering, which encourages crystallization. Flat walls have been used to induce colloidal crystallization [19,20]. Also, as predicted by Sear [21], a liquid layer on a flat wall substantially increases the rate of heterogeneous nucleation. However, the low nucleation barrier relies on the interaction between the particles and wall being translationally invariant - true for colloids but not for proteins.

Second, we have also considered crystalline surfaces. For both flat walls and pits, surfaces with lattices that are a perfect match for that of the nucleating lattice induce rapid nucleation. This match must be in both the lattice symmetry and the lattice constant. See Fig. 3(a) for a crystal that has
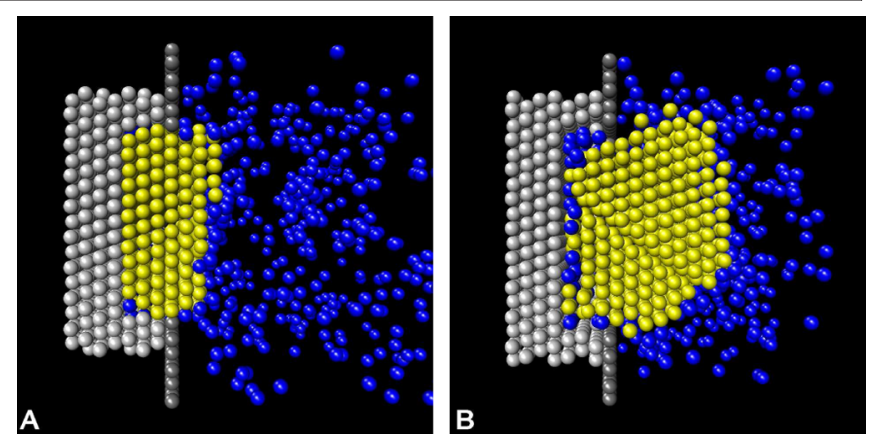

FIG. 3 (color online). Snapshots of a fluid in contact with a pit which is formed from a cylinder cut from an fcc crystal with its $\{111\}$ plane facing the fluid. The cylinders both have radius $R=$ 7 and depth $D=3$. The two panels show pores cut from fcc crystals of different densities, $\rho_{\text {wall }}$. The snapshots are cross sections through the centers of the pits. In (A) the density of the substrate crystal matches that of the nucleating crystal at coexistence, $\rho_{\text {wall }}=\rho_{\text {eq }}=0.88$. We see a defect-free crystal nucleating and growing out of the pit. In (B) the lattice constant of the substrate is $\approx 4 \%$ smaller than that of the nucleating crystal $\left(\rho_{\text {wall }}=1.0\right)$. Note the defect in the nucleating crystal caused by this mismatch in lattice constant. The dark gray, light gray, blue, and yellow particles are purely repulsive wall particles, wall particles that attract the fluid particles $\left(\epsilon_{w}=\epsilon\right)$, liquid particles, and crystalline particles, respectively.

nucleated in a shallow pit cut out of an fcc lattice, where this fcc lattice has a lattice constant very close to that of the nucleating crystal. Here the crystallization occurs rapidly. The crystalline surface is made of particles that are immobile yet otherwise identical to the fluid particles. Van Blaaderen and co-workers have observed crystallization on crystalline templates in colloidal suspensions [1].

However, even if the lattice of the surface has the same symmetry as the nucleating crystal, then even a small mismatch in lattice constant affects the result: although a crystal still nucleates, as it grows strain builds up due to the mismatch between the surface and crystal lattices. See Fig. 3(b) for a defected crystal that has formed in contact with a pit cut from an fcc crystal with a density $\approx 14 \%$ higher than that of the nucleating crystal. The resulting small mismatch in lattice constant $(\approx 4 \%)$ between the surface and nucleating crystal causes the nucleating crystal to be split by a defect. Further increasing the mismatch in lattice constant to approximately $10 \%$ prevents nucleation altogether. Clearly, any crystalline template for epitaxial growth would have to be tailor-made for a specific crystal structure of a specific protein. Such a template could never be used to crystallize many proteins.

Our findings for crystallization in the presence of a surface with nanoscale pits are summarized in Fig. 4. This is for pits with noncrystalline surfaces. The optimal region is indicated in green. In the green region of parameter space, the pits are sufficiently small that even far from the metastable liquid-liquid transition, they will fill with the concentrated protein solution phase. But they are large enough that the crystal nucleus can fit in the pit. Also, the 


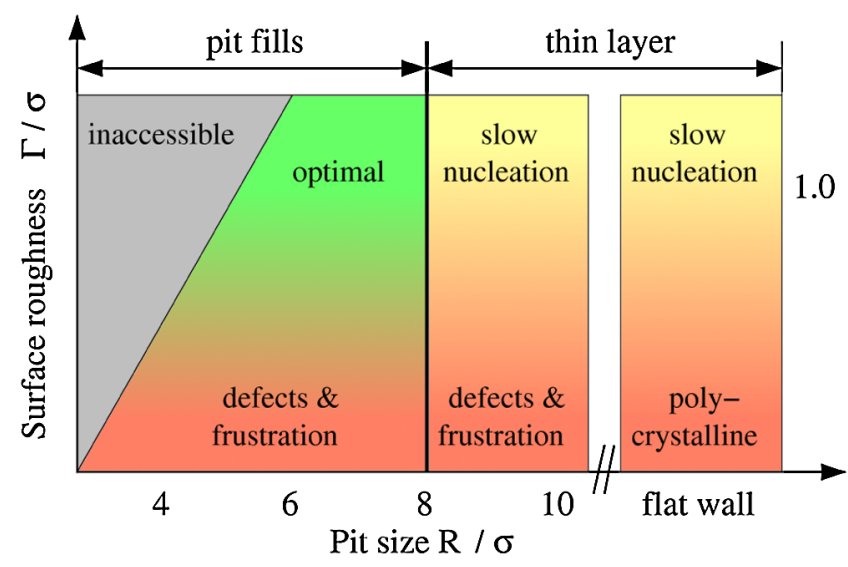

FIG. 4 (color online). Schematic that summarizes the nucleation behavior as a function of pit radius $R$ and roughness of the wall, $\Gamma$. Both are in units of the protein diameter $\sigma$. Small pits will fill with a protein liquid due to capillary condensation, and if the walls are rough, then nucleation of a crystal is rapid in this liquid. If the walls are smooth, then ordering occurs at the wall with noncrystallographic symmetry, and this inhibits crystallization.

surface of the pit is sufficiently rough that the crystal forms away from the pit surface, and so it is unstrained. Figure 4 should provide a qualitative guide for the experimental design of crystal-nucleation agents.

In conclusion, our numerical simulations reveal a mechanism for protein crystal nucleation in which a nanoscale pit fills with a droplet of the concentrated solution phase, and then a crystal nucleus forms in this confined droplet [see Fig. 1(a)]. Surprisingly, we find that it helps if the sides of the pit are rough; on smoother surfaces the crystal starts to grow in contact with the wall, but as it grows it becomes strained. This strain arises because the crystal conforms to the curved wall. The resulting build up of stress arrests growth.

Unlike epitaxial nucleation and growth, the mechanism we have found does not rely on a match between the lattice of the nucleating crystal and the lattice of the crystalline surface. Therefore, a single surface may act as a nucleant for many protein crystals. However, it does rely on the proximity of a liquid-liquid transition. George and Wilson [17] observed that many proteins crystallize when the value of their second-virial coefficient is in a range that corresponds to the solution having a liquid-liquid transition [17]. We suggest that the proteins may crystallize in this range of second-virial coefficient values because there capillary condensation can fill nanoscale pits on the surfaces of impurities in the solution. The crystallization barrier is then small in these filled pits. This capillarycondensation-assisted nucleation will happen well before homogeneous nucleation [7] can take place. As they rely only on capillary condensation, our findings should be widely applicable. Capillary condensation is a general thermodynamic phenomenon [10] that is independent of the details of the interaction potential. Therefore, it will occur in protein and other solutions but also in, for example, water. Snow frequently forms in the Earth's atmosphere under conditions where metastable liquid water can form [22], so ice nuclei may be forming in water-filled pits of hydrophilic aerosol particles.

The authors thank S. Abeln, A. Vila Verde, and K. Shundyak for fruitful discussions. The work of the FOM Institute is made possible by financial support from the Netherlands Organization for Scientific Research (NWO). D.F. acknowledges financial support from the Royal Society of London and from the ERC (Advanced Grant Agreement No. 227758). J. A. v. M. and D. F. acknowledge support from Senter Novem/Unilever.

[1] A. van Blaaderen, R. Ruel, and P. Wiltzius, Nature (London) 385, 321 (1997).

[2] N.E. Chayen, E. Saridakis, R. El-Bahar, and Y. Nemirovsky, J. Mol. Biol. 312, 591 (2001).

[3] N.E. Chayen, E. Saridakis, and R. P. Sear, Proc. Natl. Acad. Sci. U.S.A. 103, 597 (2006).

[4] P. Asanithi et al., ACS Appl. Mat. Int. 1, 1203 (2009).

[5] V. M. Bolanos-Garcia and N.E. Chayen, Prog. Biophys. Molec. Biol. 101, 3 (2009).

[6] J.D. Gunton, A. Shiryayev, and D. L. Pagan, Protein Condensation: Kinetic Pathways to Crystallization and Disease (Cambridge University Press, Cambridge, England, 2007).

[7] P. R. ten Wolde and D. Frenkel, Science 277, 1975 (1997).

[8] M. Muschol and F. Rosenberger, J. Chem. Phys. 107, 1953 (1997).

[9] D. Costa, P. Ballone, and C. Caccamo, J. Chem. Phys. 116, 3327 (2002).

[10] R. Evans, J. Phys. Condens. Matter 2, 8989 (1990).

[11] D. Frenkel and B. Smit, Understanding Molecular Simulation (Academic Press, New York, 2002).

[12] P. R. ten Wolde, M. J. Ruiz-Montero, and D. Frenkel, Faraday Discuss. 104, 93 (1996).

[13] The neighbor distance cutoff is $r_{c}=1.35$. The correlator threshold to form a link is $\vec{q}_{6}(i) \cdot \vec{q}_{6}(j)>0.55$. The number of links to form a solidlike particle is $n_{l}(i)>7$.

[14] A. J. Page and R. P. Sear, Phys. Rev. Lett. 97, 065701 (2006).

[15] D. F. Rosenbaum, P. C. Zamora, and C. F. Zukoski, Phys. Rev. Lett. 76, 150 (1996).

[16] O. Galkin and P. G. Vekilov, Proc. Natl. Acad. Sci. U.S.A. 97, 6277 (2000).

[17] A. George and W. W. Wilson, Acta Crystallogr. Sect. D 50, 361 (1994).

[18] J. A. van Meel, Ph.D. thesis, University of Amsterdam, 2009.

[19] A. D. Dinsmore, A. G. Yodh, and D. J. Pine, Phys. Rev. E 52, 4045 (1995).

[20] S. Auer and D. Frenkel, Phys. Rev. Lett. 91, 015703 (2003).

[21] R. P. Sear, J. Phys. Condens. Matter 14, 3693 (2002).

[22] J.H. Seinfeld and S. N. Pandis, Atmospheric Chemistry and Physics: From Air Pollution to Climate Change (Wiley Blackwell, 1994), 2nd ed. 